\title{
THE DISC TECHNIQUE FOR DETERMINING SENSITIVITY TO THE ANTIBIOTICS
}

\author{
BY \\ R. W. FAIRBROTHER AND G. MARTYN \\ From the Department of Clinical Pathology, Manchester Royal Infimary
}

(RECEIVED FOR PUBLICATION APRIL 16, 1951)

The range of antibiotics available for therapy is extending steadily, and in consequence there is an increasing demand on the services of the laboratory. Chloramphenicol has now been added to the free list, aureomycin is being used for certain prescribed conditions, and terramycin is generally available in the U.S.A. This form of therapy should be subjected to bacteriological control, and it is therefore important to have a simple and reliable method of determining sensitivity.

Various methods are used for these tests, and the serial dilution and the disc techniques are probably the most popular. The serial dilution method is reliable, but it is time-consuming and consequently is of limited value as a routine test.

The disc technique is now widely used for routine purposes, as a quick result can be obtained. This is a simple adaptation of the original cylinder method, in which, it should be appreciated, the assays were made at least in duplicate under carefully controlled conditions. The demand for quick results has produced a variety of disc methods, some of which have little, apart from simplicity, to offer. It should, however, be accepted that, unless a consistent standard technique is employed, the results may be very misleading. This is a matter of considerable importance if the tests are used for therapeutic control.

There are many sources of error in the disc technique, and irregular results are not uncommon. The importance of the depth of agar in the assay plates has been discussed by Hayes (1945). Some other factors are now considered.

\section{Nature of the Disc}

Two methods of using the filter-paper disc are practised, "wet" and " dry." The dried disc technique has many advantages over the wet method, in which the antibiotic may be added to the disc at the time of assay in a variety of ways, some of which are far from reliable. Dried discs can be readily prepared to a standard size and impregnated with a constant amount of antibiotic; they are easily manipulated; they can be kept for several weeks without loss of potency, but weekly control tests should be made. The test is simple and produces consistent results provided a satisfactory technique is used (Figs. $1 a$ and $b$ ). 


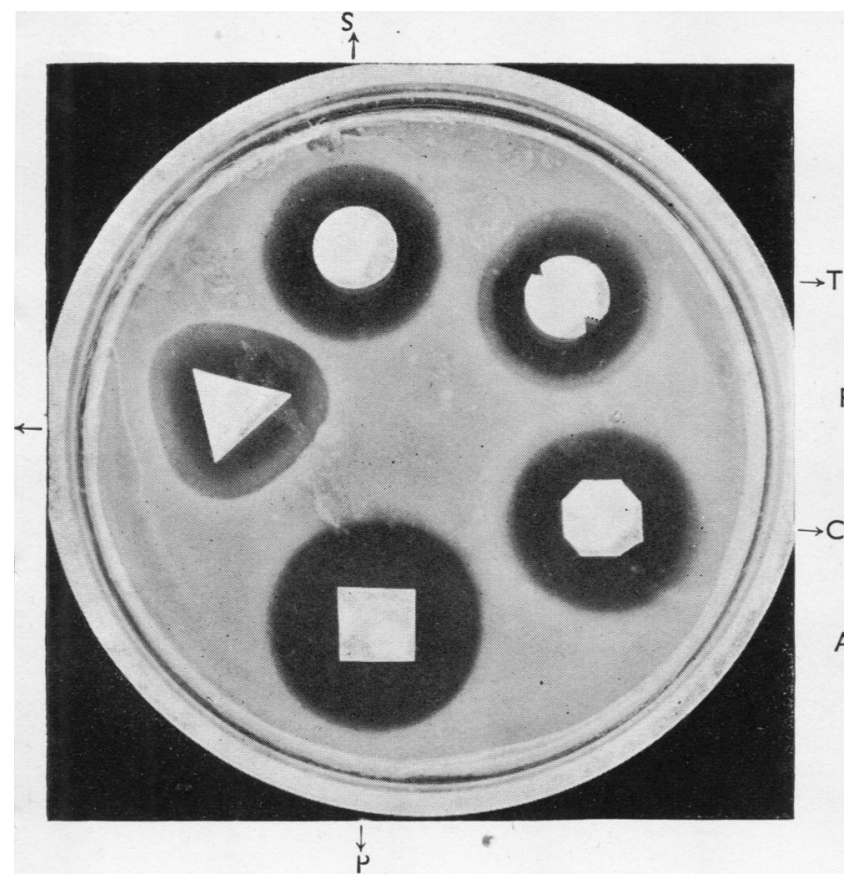

FIG. 1a.-Staph. pyogenes with standard discs (on agar). Incubated for 24 hours at $37^{\circ} \mathrm{C}$.; note development of secondary growth around A disc.

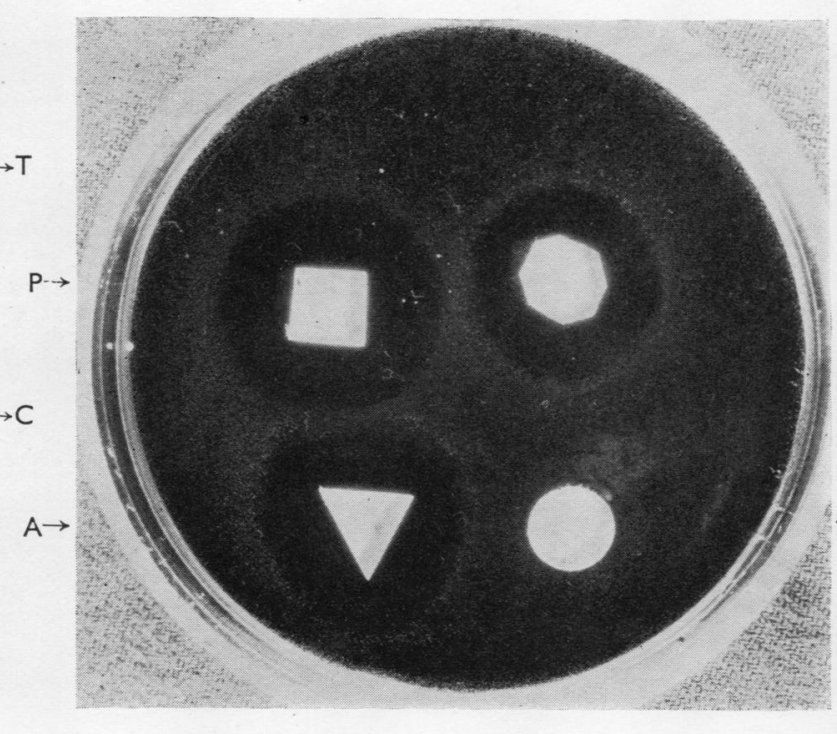

Fig. 1b.-Strept. haemolyticus (Group A) on blood-agar with standard discs.

$\mathbf{P}=$ penicillin (1.5 units), $\mathrm{A}=$ aureomycin $(10 \mu \mathrm{g}),. \mathrm{S}=$ streptomycin $(33 \mu \mathrm{g}),. \mathrm{T}=$ terramycin $(10 \mu \mathrm{g}$.$) , and \mathrm{C}=$ chloramphenicol $(33 \mu \mathrm{g}$.$) .$

\section{Strength of the Antibiotic}

It is important that the amount of antibiotic used in the tests should be adjusted so that zones of inhibition are given only by organisms whose sensitivity is within therapeutic limits. Thompson (1950) used dried filter-paper discs (Postlip Mills No. 633 or Green's No. 401) and recommended concentrations of (per ml.) penicillin, 1,000 units, streptomycin, 10,000 $\mathrm{g}$., aureomycin, 2,500 $\mu \mathrm{g}$., and chloramphenicol (chloromycetin), 2,500 $\mu \mathrm{g}$.; one drop $(0.02 \mathrm{ml}$.) was added to each disc. He claimed that the zones gave a fairly accurate guide to the sensitivity of the organisms and to the adequacy of the antibiotic dosage. These concentrations are, however, greater than those generally used in the wet disc technique.

Tests have been carried out to determine the effect on the final result of varying the concentration of antibiotic on the discs. As the amount of antibiotics added to each disc is carefully controlled, it appeared an advantage to indicate the actual amount of antibiotic on the individual discs rather than the strength per ml. of the initial solutions. The quantity added to the discs varies in different laboratories; in some a drop is added by means of a pipette or platinum loop, in others the discs are soaked in the antibiotic solutions. Therefore, while the strength of the original solutions might be the same, the final concentrations on the discs might vary considerably.

Standard discs (Whatman No. 1 or Postlip Mills No. 633 filter-paper) were sterilized in the hot air oven, impregnated with varying strengths of the antibiotics by the addition of a standard drop, and then dried in the incubator. Different shapes were used for the various antibiotics, but the size was approximately the same. The following amounts per disc were found to give results of practical value: penicillin 1.5 unit, streptomycin $33 \mu \mathrm{g}$, 
aureomycin $10 \mu \mathrm{g}$., terramycin $10 \mu \mathrm{g}$., and chloramphenicol (chloromycetin) $33 \mu \mathrm{g}$. These were prepared by adding to each disc one drop $(0.033 \mathrm{ml}$.) of the following strengths: penicillin $45 \mathrm{u}$. $/ \mathrm{ml}$., streptomycin $1,000 \mu \mathrm{g}$. $/ \mathrm{ml}$., aureomycin $300 \mu \mathrm{g}$. ml., terramycin $300 \mu \mathrm{g}$, and chloromycetin $1,000 \mu \mathrm{g} . / \mathrm{ml}$. (Fig. 1).*

There was an appreciable margin of error; a twofold increase or decrease did not significantly affect the results, but the use of quantities greatly in excess of these values tended to produce zones of inhibition around relatively insensitive organisms (Fig. 2). Thus organisms which are relatively resistant may appear to be quite sensitive by these tests if an excessive concentration of antibiotics is used. The converse also applies.

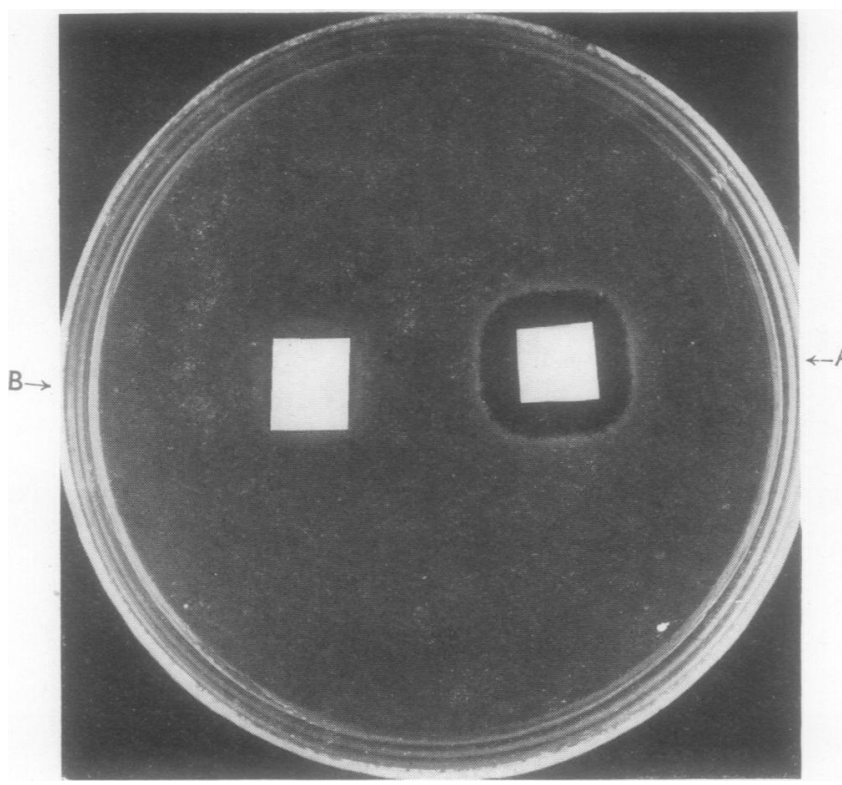

FIG. 2.-Plate seeded with Staph. pyogenes (inhibited by 6 $\mathrm{u} . / \mathrm{ml}$. in the serial dilution test) with $\mathbf{A}$ concentrated disc (33 units) and $B$ weak disc (1 unit).

Tests were made, in parallel with the serial dilution method, on a wide range of organisms. Agar or blood-agar plates were uniformly seeded, usually with a young broth culture, before the addition of the discs and finally incubated at $37^{\circ} \mathrm{C}$. for 18 hours; after this time the aureomycin discs rapidly lose potency and secondary growth occurs. The results given by the dried discs were in good agreement with those obtained by the dilution technique.

\section{Size of Inoculum}

In the original "cylinder" tests the plates were seeded by flooding the medium with a broth culture and then any excess fluid was removed; in this way a uniform confluent growth was produced. It has now become routine practice to add the discs to any form of inoculum, including original material, e.g., pus, swabs, sputum, containing one or more organisms and irrespective of the number of bacteria. It has been suggested that the size of the inoculum has no appreciable effect on the zoning (Thompson, 1950). This is contrary to our experience. It has frequently been observed, particularly with resistant staphylococci (penicillinase-producers), that zones of inhibition may be obtained with a light bacterial inoculum, i.e., one giving individual colonies, while a heavy inoculum with the same organism is quite resistant. This is illustrated in Fig. 3. It is consequently important that a standard

* Blotting-paper discs ( $8 \mathrm{~mm}$. diameter), of different colours and impregnated by means of a " 50 dropper" to give the same values, are now being used. 
inoculum should be used for the test. Flooding the medium with a young broth culture has given the best results, but heavy and even seeding from a solid medium with a platinum loop has also proved satisfactory.

In order to save time, and this is often an important consideration, the tests can sometimes be carried out directly on material collected from the patient. It is, however, important that the results should be confirmed by the use of the standard technique; this is particularly important if there is a mixed or light growth.

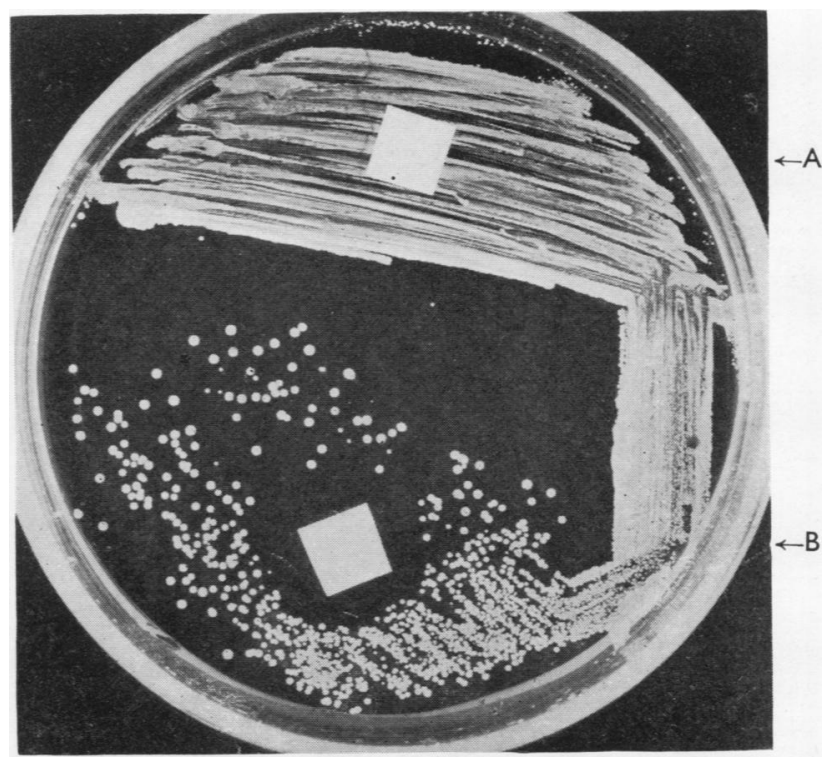

FIG. 3.-A resistant Staph. pvogenes (inhibited by $100 \mathrm{u} . / \mathrm{ml}$. in the tube method) with dried penicillin discs ( 33 units) at A heavy inoculum, no inhibition; at $B$ light inoculum, zone of inhibition indicating sensitivity.

\section{Summary}

The dried disc method is a simple, rapid, but crude method of determining sensitivity to the antibiotics. The technique must be standardized in order to minimize the inherent errors of the test; it is important to note that a uniform heavy growth is required and that the concentration of the antibiotic should be carefully controlled. Provided these requirements are fulfilled, valuable practical information of the sensitivity of an organism can be rapidly obtained. In any doubtful case, more accurate methods of assay should be carried out.

We wish to thank the Department of Clinical Photography, Manchester Royal Infirmary, for the figures, Messrs. Cyanamid Products, Ltd., for the supply of standard aureomycin hydrochloride, Messrs. Parke, Davis \& Co. for the pure chloromycetin, and the M.R.C. for the terramycin.

\section{REFERENCES}

Hayes, W. (1945). J. Path. Bact., 57, 457.

Thompson, B. A. (1950). Journal of Clinical Pathology, 3, 118. 